\title{
Cluny, 2010. Rencontres internationales
}

\section{Q OpenEdition}

10 Journals

Édition électronique

URL : https://journals.openedition.org/cem/11137

DOI : $10.4000 /$ cem. 11137

ISSN : 1954-3093

Éditeur

Centre d'études médiévales Saint-Germain d'Auxerre

Édition imprimée

Date de publication : 15 août 2009

Pagination : 355-367

ISSN : 1623-5770

Référence électronique

"Cluny, 2010. Rencontres internationales », Bulletin du centre d'études médiévales d'Auxerre | BUCEMA

[En ligne], 13 | 2009, mis en ligne le 07 septembre 2009, consulté le 22 septembre 2022. URL : http:// journals.openedition.org/cem/11137; DOl : https://doi.org/10.4000/cem.11137

Ce document a été généré automatiquement le 22 septembre 2022.

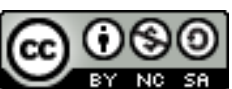

Creative Commons - Attribution - Pas d'Utilisation Commerciale - Partage dans les Mêmes Conditions 4.0 International - CC BY-NC-SA 4.0

https://creativecommons.org/licenses/by-nc-sa/4.0/ 


\section{Cluny, 2010. Rencontres internationales}

1 De nombreuses initiatives vont être prises à l'occasion du onzième centenaire de la fondation de Cluny (910-2010). Les rencontres présentées ici visent à coordonner un certain nombre de projets complémentaires élaborés par la communauté des chercheurs professionnels travaillant sur Cluny.

2 Certes, la vie scientifique n'a que faire des commémorations - même si le phénomène de "commémoration" présente un indéniable intérêt historique dans le cadre d'une étude des variations de la memoria attachée à un lieu et à une forme de vie sociale ; tel est l'objet de la rencontre qui se tiendra à Cluny les 14 et 15 mai 2010.

3 Un colloque vise, par ailleurs, à revenir sur les origines de cluny dans une étude d'ensemble de la place du monachisme au premier âge féodal (850-1050). Il est organisé en deux volets qui auront lieu, pour le premier, à Romainmôtier (Suisse) du 24 au 26 juin 2010, et pour le second à Cluny, les 9 et 10 septembre 2010.

4 En complément à ces deux initiatives, une table ronde devrait permettre de faire un point particulier sur les recherches les plus récentes consacrées au Cluny monumental, une journée grand public sera organisée sur l'actualité de la recherche clunisienne, à Cluny, le 11 septembre 2010, tandis qu'en ouverture à toutes ces manifestations, une réunion d'anthropologues et de sociologues des religions abordera la question de "Cluny dans l'histoire et la sociologie du monachisme », à Cluny du 9 au 11 avril 2010. 


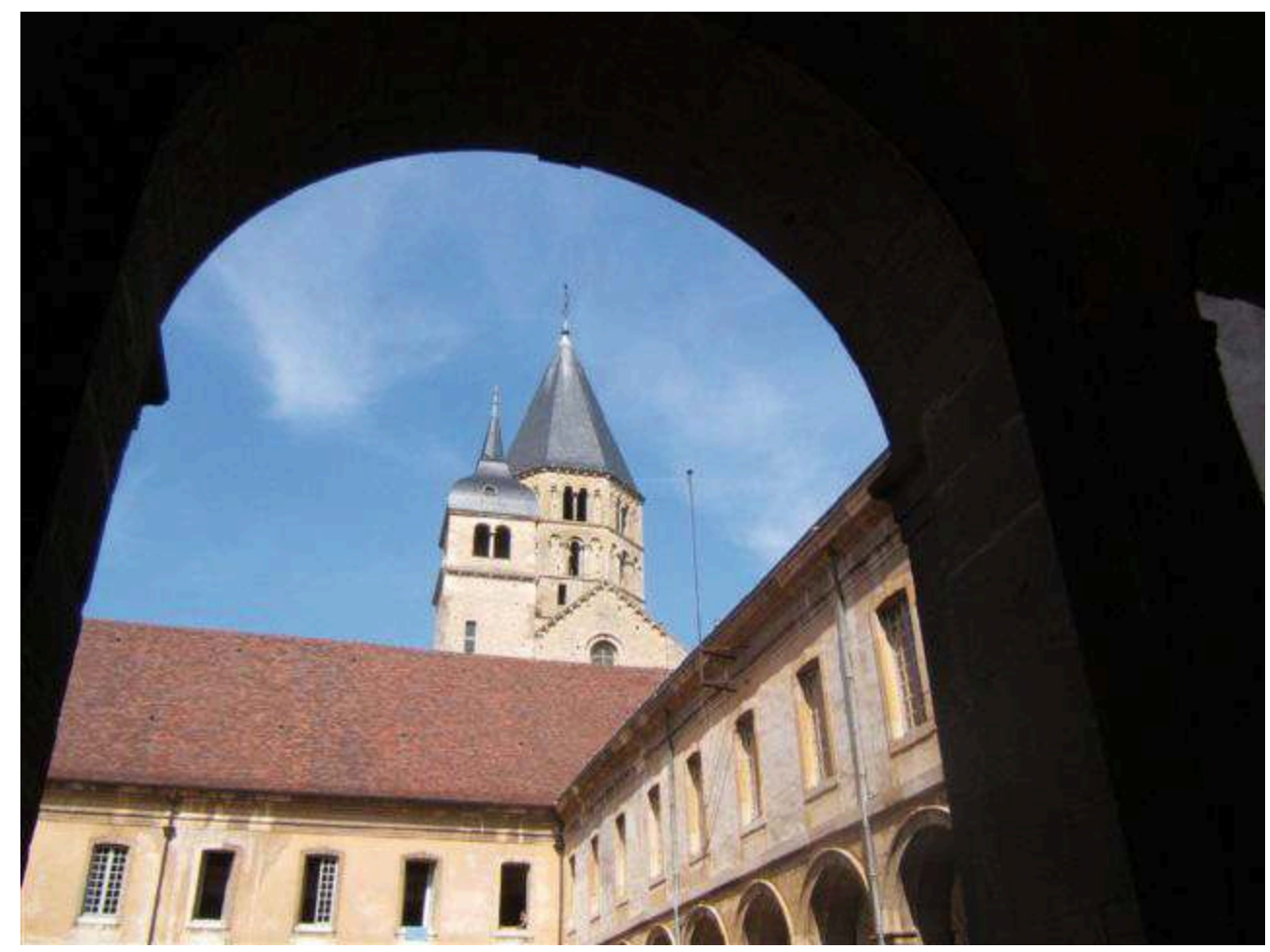

Fig. 1 - Cluny, vue du clocher depuis le cloître (cl. CEM).

\section{Comité d'organisation}

5 Anne Baud, Dominique Iogna-Prat, Eliana Magnani, Pierre-Alain Mariaux, Jean-Daniel Morerod, Isabelle Rosé, Daniel Russo et Christian Sapin

\section{Calendrier}

- Cluny, 9-11 avril 2010 - Cluny dans l'histoire et la sociologie du monachisme. Journée grand public organisée par l'Association « Cluny, chemin d'Europe » sous la direction d'Adeline Herrou et Anna Poujeau.

- Cluny, 14-15 mai 2010 - Constructions, reconstructions et commémorations clunisiennes, 1790-2010. Colloque international, organisation Didier Méhu.

- Romainmôtier, 24-26 juin 2010 - 910. Cluny, le monachisme et la société au premier âge féodal (880-1050). Partie I. Cluny, le monachisme et l'Église. Colloque international, organisation Isabelle Rosé.

- Cluny, 9-10 septembre 2010 - 910 : Cluny, le monachisme et la société au premier âge féodal (880-1050). Partie II. Cluny, le monachisme et l'émergence d'une société seigneuriale. Colloque international, organisation Florian Mazel, Michel Lauwers et Christian Sapin. - Cluny, 11 septembre 2010. Table ronde sur l'archéologie du site en 2010, sous la direction d'Anne Baud et de Christian Sapin. Actualité de l'histoire de Cluny. Journée grand public 


\section{Constructions, reconstructions et commémorations clunisiennes, 1790-2010}

Cluny, vendredi 14 et samedi 15 mai 2010

Organisation : Didier Méhu

8 En 2010, la célébration du onzième centenaire de la fondation de Cluny donnera lieu à un florilège de commémorations soutenues par les instances patrimoniales, les collectivités territoriales, le "grand public ", et dont les historiens seront tantôt les acteurs, tantôt les moteurs. Cet engouement transversal pour la commémoration d'un passé révolu laisse à réfléchir, d'autant plus qu'il apparaît au plus grand nombre comme une activité normale, louable et relevant du rôle social de l'historien. Le présent projet vise à questionner la normalité de l'activité commémorative pour la resituer dans l'histoire, celle des XIXe, XXe et du début du XXIe siècle, pour qui Cluny a pu être tantôt un objet de lamentation, d'édification, de rêve, de glorification. Au lieu de commémorer le onzième centenaire de la fondation de Cluny, nous aimerions engager une autoréflexion sur la célébration elle-même et l'objet de celle-ci, "Cluny "; objectiver ce que la demande sociale considère comme allant de soi : Cluny et son onzième centenaire.

Cette démarche implique d'analyser la manière dont on a construit les différentes facettes de l'objet «Cluny» depuis sa mort en 1790: construction d'un objet scientifique par la sauvegarde, le classement, la publication et l'étude de ses archives; construction d'un objet architectural par la plaie laissée par sa destruction inachevée, par ses restaurations, les reconstructions partielles, l'exhumation de ses débris ou de ses racines et leur transmutation en témoins archéologiques; construction d'un objet littéraire sous la plume des romanciers, des voyageurs, des historiens puis des agents du tourisme; construction d'un objet mémoriel par des célébrations répétées et par la diffusion d'une image positive du «message clunisien » pour la société contemporaine.

Cette réflexion doit impliquer les historiens, mais aussi les acteurs actuels des reconstructions clunisiennes : architectes, agents de tourisme, ingénieurs et agents de l'exécutif local. Il ne faudrait pas en effet que les célébrations de 2010 soient seulement l'occasion de présenter de nouvelles mises en valeur de Cluny, mais aussi de réfléchir aux enjeux et motivations, conscientes ou non, qui les animent.

\section{Vendredi 14 mai 2010}


3. Reconstitutions et éparpillements scientifiques

- Kathryn Brush, Arthur Kingsley Porter et la place de Cluny dans la production de l'histoire de l'art américaine

- Daniel Russo, Cluny dans l'histoire de l'art français d'Émile Mâle à Henri Focillon

\section{Samedi 15 mai 2010}

4. Reconstitutions et éparpillements scientifiques (suite)

- Isabelle Vernus, Les monumenta clunisiens : le sort des archives de Cluny

- Alain Guerreau, Les recherches sur Cluny au XXe siècle : la balkanisation et ses conséquences

- Clemens Kosch, Les constructions archéologiques de Cluny, de K.-J. Conant à A. Baud et C. Sapin

5. Reconstructions et présentations contemporaines

-Jean-Luc Delpeuch et Christian Père, Les enjeux de la reconstruction virtuelle de Cluny III

- Frédéric Didier, Les enjeux des restaurations et reconstructions monumentales de l'abbaye de Cluny

- Christophe Voros et Michel Gaudard, Les enjeux de la construction d'un réseau clunisien au XXIe siècle

- François Amy de la Bretèque, Cluny, les moines et le cinéma

18 Table ronde conclusive sous la direction de François Hartog: Commémorations, patrimoine, histoire et médiévalisme

\section{0 : Cluny, le monachisme et la société au premier âge féodal (880-1050)}

19 À l'image - mais à une autre échelle - de ce qui s'est fait en 1987, à l'occasion du millénaire capétien, ce colloque international a pour objet de réunir des médiévistes de différentes spécialités - archéologues, historiens, historiens de l'art - afin de discuter des apports historiographiques des dernières années et des recherches en cours sur les origines de Cluny replacées dans le cadre large du monachisme tardo-carolingien et pré-grégorien. Alors que seule une véritable histoire de la société peut permettre de saisir pleinement l'étendue du phénomène clunisien, il s'agit de prendre en compte toute l'importance du tournant jadis qualifié par Marc Bloch de " premier âge féodal ». Dépassant largement Cluny et le seul horizon de la Bourgogne - rôle des moines dans la confusion Église/société ; importance des usages monastiques dans la conception de l'échange ; inscription spatiale des pouvoirs; fonctionnalité des pratiques artistiques et monumentales; " scripturalité » etc. -, ces discussions seront articulées autour d'une rencontre en deux volets, qui auront lieu à Romainmôtier en juin 2010 et à Cluny en septembre 2010. Dans les deux cas, il s'agira d'étudier le monachisme comme agent de transformation sociale en un temps charnière, celui de la constitution d'une société seigneuriale caractérisée par les rapports de domination: domination des hommes, domination de la terre et contrôle des échanges. En quoi, les seigneuries ecclésiastiques comme Cluny ont-elles pu participer de ce mouvement général, constitutif du «féodalisme »? Dans quelle mesure la "sacralité » des terres de saint Pierre a-t-elle conféré à cette seigneurie, cœur d'une véritable "Église », un statut spécifique ? Telles sont les principales questions d'histoire sociale que ces deux rencontres entendent 
poser en recourant à toutes les approches possibles (culturelles, politiques, économiques), au point de rencontre entre les diverses spécialités académiques intéressées par l'histoire de Cluny: histoire, histoire de l'art, archéologie, liturgie, musicologie... Dans un contexte commémoratif, qui risque d'accentuer les travers bien connus d'une histoire des origines clunisiennes limitées aux approches "religieuses", nous voudrions persuader le grand public, invité à suivre les débats, que l'intelligence historique du "phénomène » clunisien ne peut se comprendre que dans le contexte social qui a présidé à la fondation du monastère.

\section{Romainmôtier, du jeudi 24 au samedi 26 juin 2010. I. Cluny, le monachisme et l'Église}

organisation : Anne Baud, Dominique Iogna-Prat, Eliana Magnani, Pierre-Alain Mariaux, Jean-Daniel Morerod, Isabelle Rosé, Daniel Russo et Christian Sapin

\section{Jeudi 24 juin 2010}

Isabelle Rosé, coordination

1. Le monachisme un agent de transformations pour la société et pour l'Église

- Isabelle Rosé, Le premier Cluny

- Noëlle Deflou-Leca, Églises et réseaux monastiques

2. Évêques, chanoines et moines, une pragmatique sociale différenciée

- Hélène Noizet et Charles Meriaux, Chanoines, moines et espace urbain dans le nord de la France

- Geneviève Bührer-Thierry, Modèles sociaux et rôle politique de l'épiscopat au Xe siècle

Dominique Iogna-Prat, coordination

3. «Culte» et "culture»: le monachisme réformateur tardo-carolingien et prégrégorien

3.1. Les travaux d'écriture et leurs finalités

- Dominique Iogna-Prat, Écrire pour se défendre : l'ecclésiologie des moines clunisiens entre Empire carolingien et Réforme grégorienne

- Pierre Chastang, Tradition documentaire et gestion des ressources écrites : autour des cartulaires de Cluny

- Eduardo Henrik Aubert, Les raisons des neumes : stratégies et approches de l'écriture du chant à Cluny

\subsection{Liturgie et vie au couvent, de Saint-Gall aux débuts de Cluny}

- Julian Hendrix, La liturgie dans le monachisme carolingien : historiographie des manuscrits liturgiques de Saint-Gall

- Susan Boynton, La liturgie à Cluny avant l'abbatiat d'Hugues de Semur : problématique de la recherche

Vendredi 25 juin 2010

Daniel Russo, coordination 
4. Des mondes du visuel

- Daniel Russo, Présence de l'absence. Conceptions de et sur l'art monastique aux IXe-XIe siècles

- Pierre-Alain Mariaux, La « faisabilité » d'un art par et pour les moines (IXe-XIe siècle)

4.1. Mises au point

- Barbara Franzé, Autour des décors peints dans l'architecture : l'exemple de Saint-Chef en Dauphiné

-Stefano Riccioni, Les décors à mosaïques : l'exemple romain

4.2. Débats et discussions : Pierre-Alain Mariaux, Didier Méhu et Daniel Russo

Christian Sapin, coordination

5. L'espace monumental des moines réformateurs

- Christian Sapin, Introduction sur l'espace monumental clunisien et les réformes au Xe siècle

- Eleonora de Stefanis, Le monachisme clunisien dans l'Italie du Nord

- Immaculada Lorés, Les monastères en Espagne au Xe siècle : tradition et réforme

- Hans Rudolf Sennhauser, Après Saint-Gall, l'archéologie des installations monastiques

- Anne Baud et C. Sapin, Cluny et l'organisation des bâtiments monastiques : dernières recherches

\section{Conclusions :}

- Pascual Martinez Sopena, Réforme monastique et transformation sociale?

\section{Samedi 26 juin 2010}

\section{Visite}

Romainmôtier et le patrimoine clunisien en Suisse, avec Peter Eggenberger, archéologue

\section{Cluny, du jeudi 9 au samedi 11 septembre 2010. II. Cluny, le monachisme et l'émergence d'une société seigneuriale}

organisation : Anne Baud, Dominique Iogna-Prat, Eliana Magnani, Pierre-Alain Mariaux, Jean-Daniel Morerod, Isabelle Rosé, Daniel Russo et Christian Sapin

\section{Jeudi 9 septembre 2010}

\section{L'émergence d'une société seigneuriale}

- Régine Le Jan, Qu'est-ce que le «premier âge féodal »?

- Stefen Patzold, Le premier âge féodal a-t-il un sens en Germanie?

- Jean-Daniel Morerod, Le premier âge féodal vu de Bourgogne (à confirmer)

\section{Florian Mazel et Michel Lauwers, coordination}

2. Les moines, la seigneurie, l'encadrement des hommes au premier âge féodal

2.1. Perspectives générales

- Eliana Magnani, Les moines et les circuits de l'« échange »

- Jean-Pierre Devroey, Du grand domaine carolingien à la seigneurie monastique

- Michel Lauwers, Les moines grands seigneurs entre incastellamento et inecclesiamento 


\section{Vendredi 10 septembre 2010}

\subsection{Approche régionale}

- Florian Mazel, Moines, clercs, seigneurs dans l'ancienne province de Tours

- Laurent Ripart, Église et société dans la moyenne vallée du Rhône, 950-1050

- Laurent Schneider, Autour de Saint-Sauveur d'Aniane : la territorialisation des pouvoirs et l'encadrement de l'habitat à l'échelle locale

- Hélène Debax, Serments, châteaux et fiefs : la spatialisation des seigneuries entre Pyrénées et Montagne Noire (Xe-XIe siècles)

\section{Conclusions}

- Barbara H. Rosenwein, Un premier âge féodal monastique?

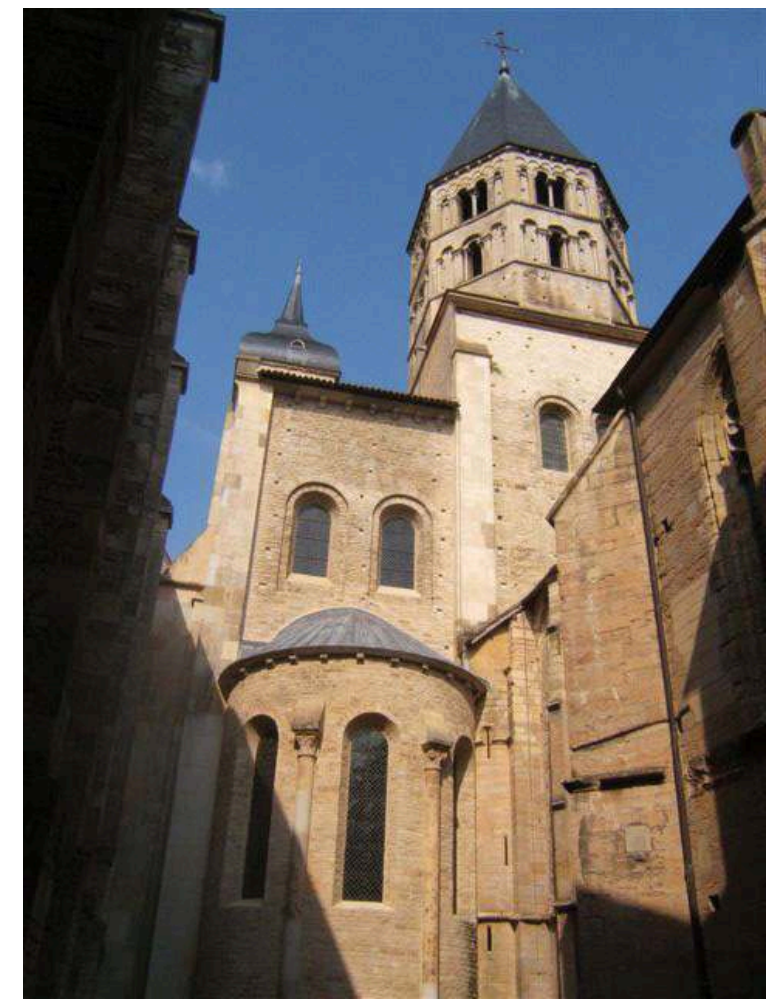

Fig. 2 - Cluny, vue du transept depuis l'est (cl. CEM). 


\section{Samedi 11 septembre 2010}

Table ronde coordonnée par Anne Baud et Christian Sapin, Les nouvelles données archéologiques. Les fouilles de Cluny (2007-2009) et leurs enjeux scientifiques pour Cluny I et Cluny II

Présentation en salle et sur le terrain, avec la participation de Charles Bonnet, JeanPierre Caillet, Jordi Camps, Gisella Cantino-Wataghin, Federico Marazzi, Hans-Rudolf Seenhauser, Neil Stratford, Éliane Vergnolle, Clemens Kosch et Étienne louis

Soirée et dimanche : conférences grand public et visite des fouilles

- Giles Constable, Cluny, 910

- Neil Stratford, Cluny et l'histoire de l'art

- Franz Neiske, Commémorer à Cluny

- Denyse Riche et Daniel-Odon Hurel, «Cluny, après Cluny »

\section{Supports institutionnels}

-ARTeHIS - UMR 5594 (Dijon), CNRS/université de Bourgogne (Dijon)

- Centre d'études médiévales d'Auxerre

- Université de Neuchâtel

- Avec la collaboration du LAMOP (Paris I/Panthéon-Sorbonne), du Cerhio (Rennes II), et le soutien de la Fédération des sites clunisiens.

\section{Participants}

- Claudie Amado (Aix-en-Provence)

- François Amy de la Bretèque (université de Montpellier III)

- Eduardo Henrik Aubert (Ehess, Gahom, Paris)

- Geneviève Bührer-Thierry (université de Marne-la-Vallée)

- Charles Bonnet (Genève)

- Susan Boynton (Columbia University, New York)

- Luc Bourgeois (université de Poitiers)

- Kathryn Brush (London, ON, University of Western Ontario)

- Jean-Pierre Caillet (université Sorbonne-Paris IV)

- Jordi Camps (Barcelone)

- Gisella Cantino-Wataghin (université de Vercelli)

- Pierre Chastang (université de Versailles-Saint-Quentin-en-Yvelines)

- Giles Constable (Princeton)

- Hélène Debax (Toulouse)

- Noëlle Deflou-Leca (université de Grenoble)

- Jean-Luc Delpeuch (ville de Cluny)

- Frédéric Didier (Monuments historiques, Paris)

- Jean-Pierre Devroey (université libre de Bruxelles)

- Peter Eggenberger (archéologue)

- Élizabeth Emery (Montclair State University, New Jersey)

- Barbara Franzé (Genève)

- Michel Gaudard (Fédération des sites clunisiens, Romainmôtier)

- Alain Guerreau (CNRS, Paris) 
- François Hartog (EHESS, Paris)

- Julian Hendrix (University of Tennessee, Knoxville, Usa)

- Adeline Herrou (CNRS/université de Paris X-Nanterre)

- Daniel-Odon Hurel (Saint-Étienne)

- Dominique Iogna-Prat (LAMOP, CNRS/université de Paris I-Panthéon Sorbonne)

- Clemens Kosch (Landesmuseum für Kloster Kultur, Lichtenau)

- Michel Lauwers (Université de Nice-Sophia Antipolis)

- Régine Le Jan (Université de Paris I-Panthéon Sorbonne)

- Immaculada Lorés (université de Lleida)

- Étienne Louis (agglomération du Douaisis)

- Eliana Magnani (CNRS, UMR 5594 ARTeHIS-Auxerre/Dijon)

- Federico Marazzi (Naples)

- Janet Marquardt (Eastern Illinois University, Charleston)

- Pascual Martinez Sopena (Universidad de Valladolid)

- Florian Mazel (université de Rennes II)

- Charles Meriaux (université Charles-de-Gaulle Lille III)

- Didier Méhu (université de Laval, Québec)

- Jean-Daniel Morerod (Université de Neuchâtel)

- Franz Neiske (Münster)

- Hélène Noizet (université de Paris I-Panthéon Sorbonne)

- Anne Nyssen-Jaubert (université de Tours)

- Stefen Patzold, (Tübingen)

- Christian Père (Ensam, Cluny)

- Yann Potin (université Paris I-Panthéon Sorbonne)

- Anna Poujeau (CNRS/université de Paris X-Nanterre)

- Daniel Prigent (Service départemental de Maine-et-Loire)

- Alain Rauwel (université de Bourgogne, UMR 5594 ARTeHIS)

- Stefano Riccioni (École normale supérieure de Pïse)

- Denyse Riche (université de Lyon II)

- Isabelle Rosé (université de Rennes II)

- Barbara H. Rosenwein (Loyola University, Chicago)

- Daniel Russo (université de Bourgogne, UMR 5594 ARTeHIS ; IUF)

- Christian Sapin (CNRS, UMR 5594 ARTeHIS - Auxerre/Dijon)

- Laurent Schneider (CNRS, UMR 6572 Lamm, Aix-en-Provence)

- Hans-Rudolf Sennhauser (Zursach)

- Eleonora de Stefanis (université de Vercelli)

- Neil Stratford (Londres)

- Éliane Vergnolle (Paris)

- Isabelle Vernus (Archives départementales de Saône-et-Loire)

- Christophe Voros (Fédération des sites clunisiens, Cluny) 


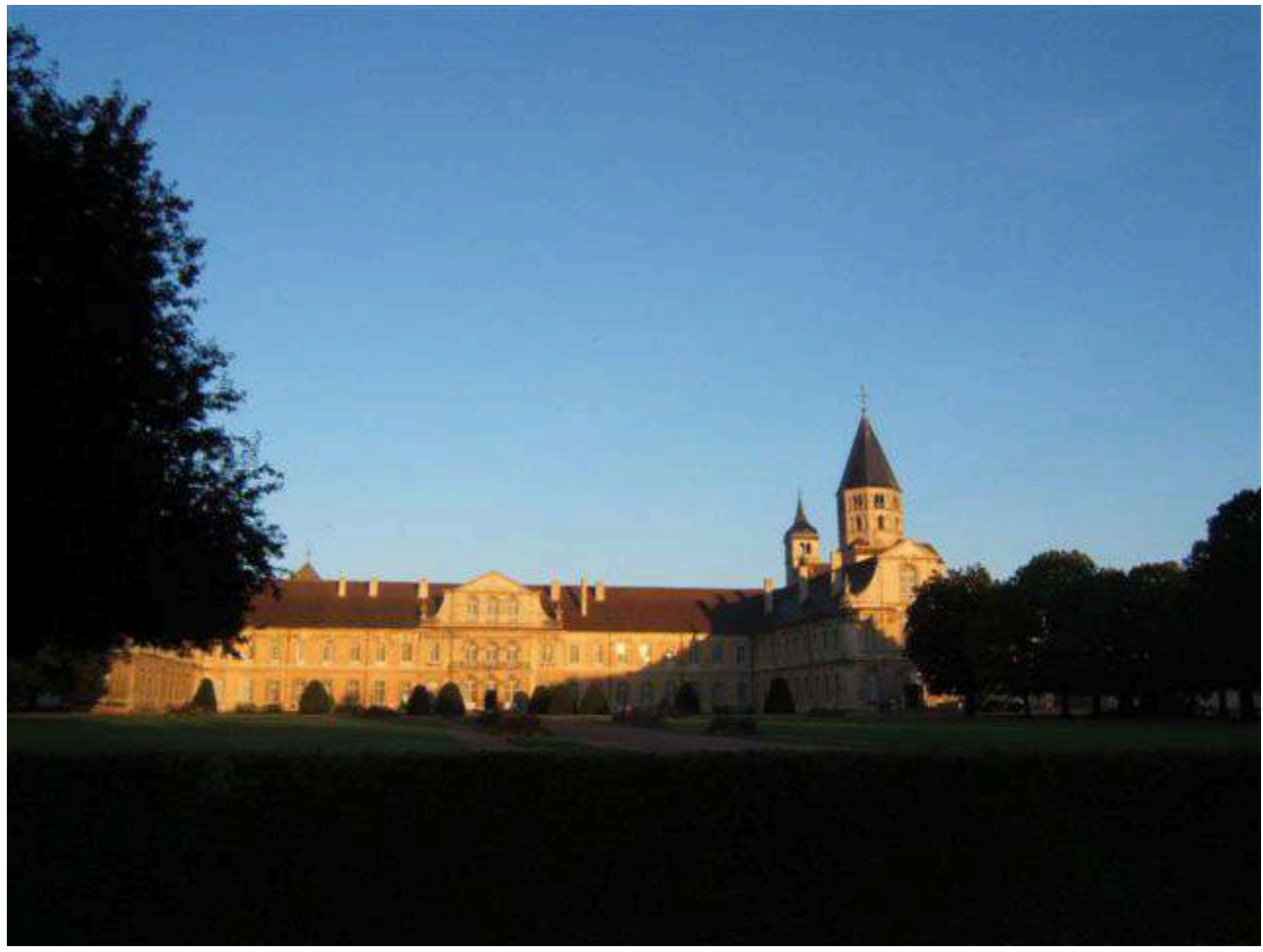

Fig. 3 - Cluny, vue générale des bâtiments du XVIIle siècle depuis l'est (cl. CEM). 